\title{
Role of spd- Continuum Components in the Halo Nucleus ${ }^{6} \mathrm{He}$
}

\author{
Jagjit $\operatorname{Singh}^{1,2, a)}$ \\ ${ }^{1}$ Dipartimento di Fisica e Astronomia “G.Galilei”, via Marzolo 8,I-35131 Padova, Italy \\ ${ }^{2}$ INFN-Sezione di Padova, via Marzolo 8, I-35131 Padova, Italy. \\ a) Corresponding author: jsingh@pd.infn.it
}

\begin{abstract}
Role of different continuum components in the weakly bound nucleus ${ }^{6} \mathrm{He}$ is studied by coupling unbound spd-waves of ${ }^{5} \mathrm{He}$ by using simple pairing contact-delta interaction. The results show that ${ }^{6} \mathrm{He}$ ground state $0^{+}$displays collective nature by taking contribution from five different oscillating continuum states that sum up to give an exponentially decaying bound wavefunction emerging from five different possible configurations i.e. $\left(s_{1 / 2}\right)^{2},\left(p_{1 / 2}\right)^{2},\left(p_{3 / 2}\right)^{2},\left(d_{3 / 2}\right)^{2}$ and $\left(d_{5 / 2}\right)^{2}$. The ground state properties of ${ }^{6} \mathrm{He}$ has been calculated.
\end{abstract}

\section{INTRODUCTION}

The nucleus ${ }^{6} \mathrm{He}$ is a typical "neutron skin or halo"[1] with a very low neutron pair separation energy $(0.975 \mathrm{MeV})$ [2]. Motivated by the recent experimental measurements at GANIL [3] on continuum resonances in ${ }^{6} \mathrm{He}$, recently we have developed a simple theoretical model [4] to study the weakly bound ground state and low-lying continuum states of ${ }^{6} \mathrm{He}$ by coupling two unbound p-waves of ${ }^{5} \mathrm{He}$. In the present study we have extended the model space with inclusion of sd- continuum waves of ${ }^{5} \mathrm{He}$. The large basis set of these spd- continuum wavefunctions are used to construct the two-particle ${ }^{6} \mathrm{He}$ ground state (g.s.) $0^{+}$emerging from five different possible configurations i.e. $\left(s_{1 / 2}\right)^{2}$, $\left(p_{1 / 2}\right)^{2},\left(p_{3 / 2}\right)^{2},\left(d_{3 / 2}\right)^{2}$ and $\left(d_{5 / 2}\right)^{2}$. The simple pairing contact-delta interaction is used and pairing strength is adjusted to reproduce the bound ground state of ${ }^{6} \mathrm{He}$. The extension of model space is a computationally challenging problem. The main aim is to show how an extension of theoretical concepts related to residual interactions, namely a contact delta pairing interaction, naturally explain the stable character of the bound states of Borromean nuclei, such as ${ }^{6} \mathrm{He}$ and simultaneously account for some of the resonant structures seen in the low-lying energy continuum.

\section{SPECTRUM OF ${ }^{5} \mathrm{He}$}

The unbound nucleus ${ }^{5} \mathrm{He}$ can be described as an inert ${ }^{4} \mathrm{He}$ core with an unbound neutron moving in $p$ doublet or $d$ doublet or $s$ singlet in simple independent-particle shell model picture. These doublets are further splitted by spin-orbit interaction. Experimentally only the $p_{3 / 2}$ and $p_{1 / 2}$ resonances are confirmed at 0.789 and $1.27 \mathrm{MeV}$ above the neutron separation threshold and their widths are quoted as $0.648 \mathrm{MeV}$ and $5.57 \mathrm{MeV}$ respectively [5]. Theoretically in order to extend the model space we have also included the $s d$-shell in picture. The continuum monopole $(\ell=0)$, dipole $(\ell=1)$ and quadrupole $(\ell=2)$ scattering single particle states $\left(E_{C}>0, k>0\right)$ of ${ }^{5} \mathrm{He}$ (see Fig- $\left.(1)\right)$ are generated with Woods-Saxon (WS) potential given by

$$
V_{W S}=\left[V_{0}+V_{l s} r_{0}^{2}(\vec{l} \cdot \vec{s}) \frac{1}{r} \frac{d}{d r}\right]\left[1+\exp \left(\frac{r_{0}-r}{a}\right)\right]^{-1}
$$

where $r=r_{0} A^{\frac{1}{3}}$. For ${ }^{5} \mathrm{He}$ the parameter set used is WS potential depth $V_{0}=-42.6 \mathrm{MeV}, r_{0}=1.2 \mathrm{fm}, a=0.9 \mathrm{fm}$ and spin-orbit coefficient $V_{l s}=8.5 \mathrm{MeV}$. 


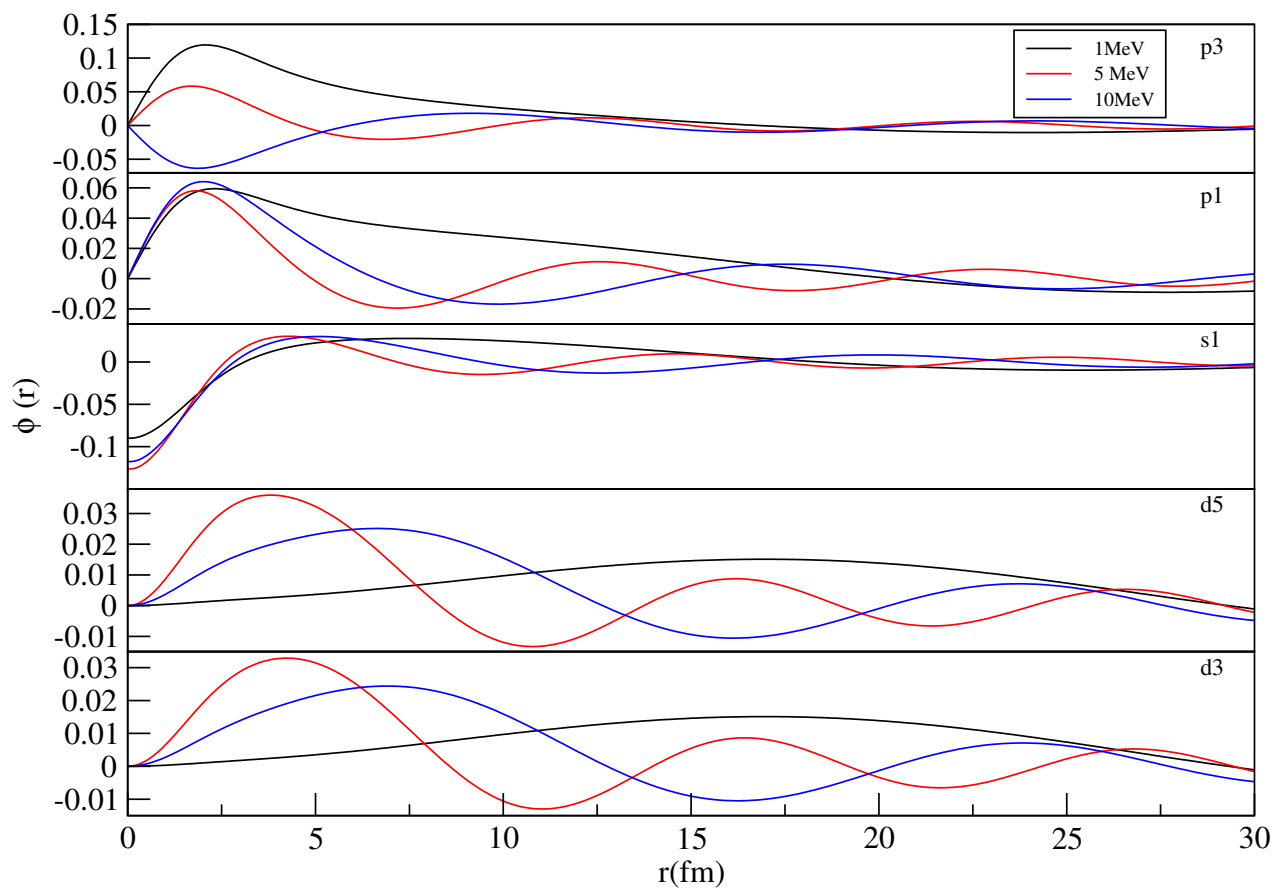

FIGURE 1: (Color online) ${ }^{5} \mathrm{He}$ continuum waves as a function of radial variable for continuum energies 1,5 and 10 $\mathrm{MeV}$ respectively

\section{MODEL AND RESULTS}

The simple model with two non-interacting particles in the above single-particle levels of ${ }^{5} \mathrm{He}$ produces different parity states (see TABLE-1) when two neutrons are placed in five different $s_{1 / 2}, p_{1 / 2}, p_{3 / 2}, d_{3 / 2}$ and $d_{5 / 2}$ unbound orbits. Namely five configurations $\left(s_{1 / 2}\right)^{2},\left(p_{1 / 2}\right)^{2},\left(p_{3 / 2}\right)^{2},\left(d_{3 / 2}\right)^{2}$ and $\left(d_{5 / 2}\right)^{2}$ couple to $J=0^{+}$.

TABLE 1: Possible configurations of ${ }^{6} \mathrm{He}$ arising from two neutrons in s-, p- and d-orbitals

\begin{tabular}{llllll}
\hline & $s_{1 / 2}$ & $p_{1 / 2}$ & $p_{3 / 2}$ & $d_{3 / 2}$ & $d_{5 / 2}$ \\
\hline$s_{1 / 2}$ & $0^{+}$ & $0^{-}, 1^{-}$ & $1^{-}, 2^{-}$ & $1^{+}, 2^{+}$ & $2^{+}, 3^{+}$ \\
$p_{1 / 2}$ & & $0^{+}$ & $1^{+}, 2^{+}$ & $1^{-}, 2^{-}$ & $2^{-}, 3^{-}$ \\
$p_{3 / 2}$ & & & $0^{+}, 2^{+}$ & $0^{-}, 1^{-}, 2^{-}, 3^{-}$ & $1^{-}, 2^{-}, 3^{-}, 4^{-}$ \\
$d_{3 / 2}$ & & & & $0^{+}, 2^{+}$ & $1^{+}, 2^{+}, 3^{+}, 4^{+}$ \\
$d_{5 / 2}$ & & & & & $0^{+}, 2^{+}, 4^{+}$ \\
\hline
\end{tabular}

The two-particle wave functions are constructed by tensor coupling of two continuum states of ${ }^{5} \mathrm{He}$. The five states discussed above are not discrete, but rather depend on the energies of the continuum orbitals. Each single particle continuum wavefunction is given by

$$
\phi_{\ell, j, m}\left(\vec{r}, E_{C}\right)=\phi_{\ell, j}\left(r, E_{C}\right)\left[Y_{\ell m_{\ell}}(\Omega) \times \chi_{1 / 2, m_{s}}\right]_{m}^{(j)}
$$

The combined tensor product of these two is given by

$$
\psi_{J M}\left(\vec{r}_{1}, \vec{r}_{2}\right)=\left[\phi_{\ell_{1}, j_{1}, m_{1}}\left(\vec{r}_{1}, E_{C 1}\right) \times \phi_{\ell_{2}, j_{2}, m_{2}}\left(\vec{r}_{2}, E_{C 2}\right)\right]_{M}^{(J)}
$$

For simplicity an attractive pairing contact delta interaction $\left(-g \delta\left(\vec{r}_{1}-\vec{r}_{2}\right)\right)$ is used, because we can reach the goal with only one parameter adjustment. The continuum single-particle wavefunctions are calculated with energies from 0.0 

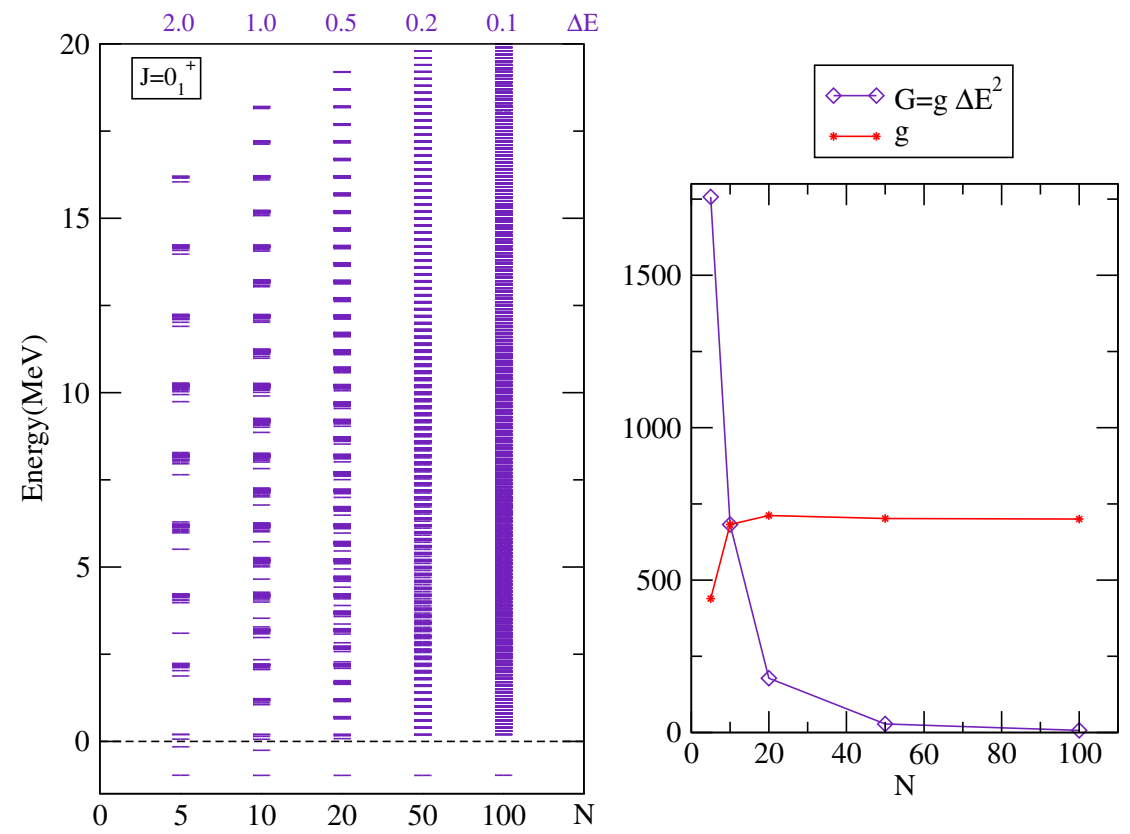

FIGURE 2: (Color online) Eigenspectrum of the interacting two-particle case for $J=0$ for increasing basis dimensions, $N$. The coefficient of the $\delta$-contact matrix, $G$, has been adjusted each time to reproduce the g.s. energy (right). The actual strength of the pairing interaction, $g$, is obtained by correcting with the energy spacing $\Delta E$ and it is practically a constant.

to $10.0 \mathrm{MeV}$ and normalized to a delta for the spd-states of ${ }^{5} \mathrm{He}$ on a radial grid which varies from $0.1 \mathrm{fm}$ to 100.0 $\mathrm{fm}$ with the potential discussed above. The two particle states are formed using mid-point method with an energy spacing of 2.0, 1.0, 0.5, 0.2 and $0.1 \mathrm{MeV}$ corresponding to block basis dimensions of $N=5,10,20,50$ and 100 respectively and the matrix elements of the pairing interaction are calculated. In Fig-2, the eigenspectrum for $J=0^{+}$ case is presented and from figure it is clear that with increase in basis dimensions the superflous bound states moves into the continuum. The coefficient of the $\delta$-contact matrix $(G)$ is adjusted to reproduce the correct ground state energy. The actual pairing interaction $g$ is obtained by correcting with a factor which depends on the aforementioned spacing between energy states. The correction factor is practically a constant quantity, except for the smallest basis. The biggest adopted basis size gives a fairly dense continuum in the region of interest. The radial part of the $S=0$ ground state wavefunction obtained from the diagonalization in the largest basis is presented in the left part of Fig3. It shows a certain degree of collectivity, taking contributions of comparable magnitude from several basis states. The surface plot shows the exponential behavior typical of a bound state, despite being the sum of many products of oscillating wavefunctions. One can see from the right part of the Fig.- 3 that the square of the amplitudes of the $\left(p_{3 / 2}\right)^{2}$ components are dominant summing up to $89.7 \%$. The comparison among present results and earlier studies by T.Myo [6] and Hagino [7] is presented in TABLE-2 and from this table it is clear that present results are in good agreement with earlier calculations, which further strengthen present results. In TABLE-3, calculated ground state properties are compared with earlier studies[6,7], where $R_{m}$ is the matter radius,

$$
\left\langle r_{N N}\right\rangle=\left\langle\psi_{g s}\left(\vec{r}_{1}, \vec{r}_{2}\right)\left|\left(\vec{r}_{1}-\vec{r}_{2}\right)^{2}\right| \psi_{g s}\left(\vec{r}_{1}, \vec{r}_{2}\right)\right\rangle
$$

is the mean square distance between the valence neutrons, and

$$
\left\langle r_{c-N N}\right\rangle=\left\langle\psi_{g s}\left(\vec{r}_{1}, \vec{r}_{2}\right)\left|\left(\vec{r}_{1}+\vec{r}_{2}\right)^{2} / 4\right| \psi_{g s}\left(\vec{r}_{1}, \vec{r}_{2}\right)\right\rangle
$$

is the mean square distance of their centre of mass with respect to the core. 

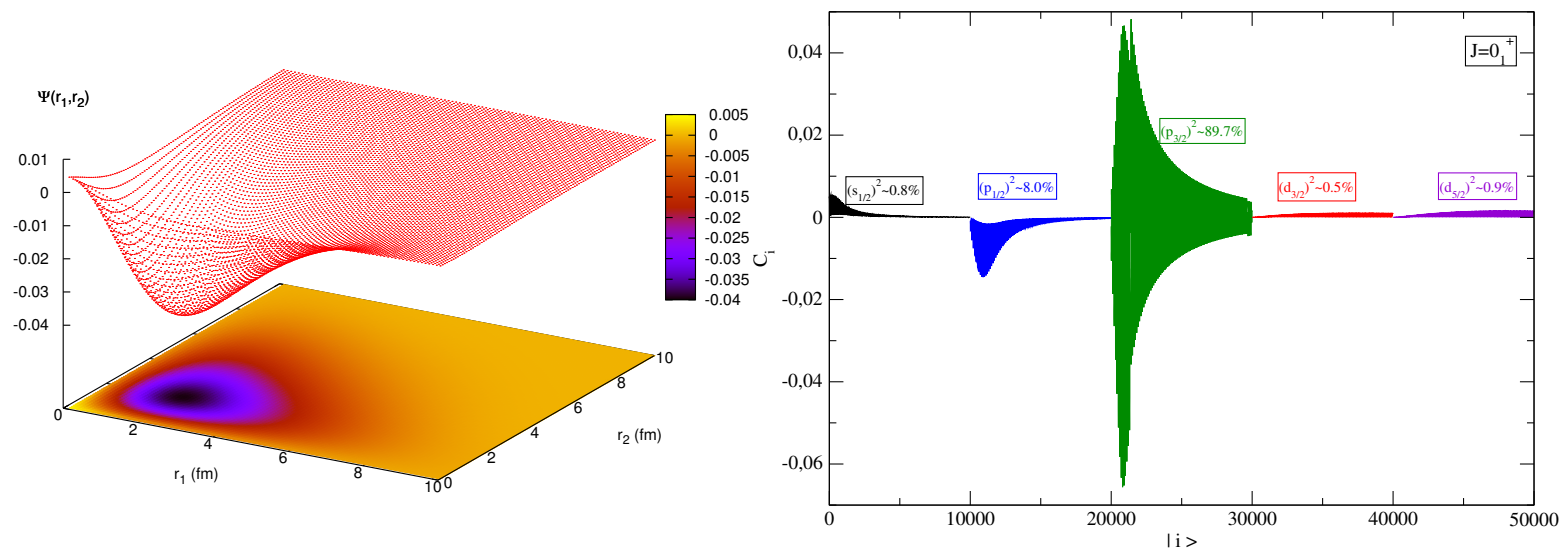

FIGURE 3: (Color online) Ground state wavefunction $(S=0)$ for $N=100$ as a function of the coordinates of the two neutrons and corresponding contour plot (Left part). Decomposition of the g.s. into the $\mathrm{J}=0$ basis (Right part) as a function of an arbitrary basis state label $\mid i>$ : the basis is divided into five blocks, $10^{4}\left[s_{1 / 2} \times s_{1 / 2}\right]^{(0)}, 10^{4}\left[p_{1 / 2} \times p_{1 / 2}\right]^{(0)}$, $10^{4}\left[p_{3 / 2} \times p_{3 / 2}\right]^{(0)}, 10^{4}\left[d_{3 / 2} \times d_{3 / 2}\right]^{(0)}$ and $10^{4}\left[d_{5 / 2} \times d_{5 / 2}\right]^{(0)}$ components.. The ordering in each block is established by the sequential energies of each pair of continuum s.p. states, i.e. $\left(E_{C_{1}}, E_{C_{2}}\right)=(0.1,0.1),(0.1,0.2), \ldots,(0.1,10.0)$, $(0.2,0.1),(0.2,0.2), \ldots(10.0,10.0)$.

\begin{tabular}{cccc}
\hline Config. & Present & T.Myo[6] & Hagino[7] \\
\hline$\left(2 s_{1 / 2}\right)^{2}$ & 0.008 & 0.009 & - \\
$\left(1 p_{1 / 2}\right)^{2}$ & 0.080 & 0.043 & - \\
$\left(1 p_{3 / 2}\right)^{2}$ & 0.897 & 0.917 & 0.830 \\
$\left(1 d_{3 / 2}\right)^{2}$ & 0.005 & 0.007 & - \\
$\left(1 d_{5 / 2}\right)^{2}$ & 0.009 & 0.024 & - \\
\hline
\end{tabular}

TABLE 2: Components of the ground state $\left(0_{1}^{+}\right)$ of ${ }^{6} \mathrm{He}$

\begin{tabular}{cccc}
\hline & Present & T.Myo[6] & Hagino[7] \\
\hline$R_{m}$ & 2.37674 & 2.37 & $\ldots$ \\
$r_{N N}^{2}$ & 28.8404 & 23.2324 & 21.3 \\
$r_{c-2 N}^{2}$ & 7.21011 & 9.9225 & 13.2 \\
\hline
\end{tabular}

TABLE 3: Radial properties of the ground state of ${ }^{6} \mathrm{He}$ in units of fm

\section{CONCLUSIONS}

In the present study I present emergence of bound halo ground state of ${ }^{6} \mathrm{He}$ from the coupling of five unbound spdwaves in the continuum of ${ }^{5} \mathrm{He}$, due to presence of pairing interaction. Contribution of different configurations has been presented. Radial properties of ground state of ${ }^{6} \mathrm{He}$ are also presented and compared with other calculations.

\section{ACKNOWLEDGMENTS}

The useful discussions with L.Fortunato, A.Vitturi, R.Chatterjee and Sukhjeet Singh and financial assistance from Fondazione Cassa di Risparmio di Padova e Rovigo (CARIPARO) is gratefully acknowledged.

\section{REFERENCES}

[1] I. Tanihata et al., Physics Letters B 160, 380 - 384 (1985).

[2] F. Ajzenberg-Selove, Nucl. Phys. A 490, 1 - 225 (1988).

[3] X.Mougeot et al., Physics Letters B 718, $441-446$ (2012).

[4] L.Fortunato, R.Chatterjee, J. Singh, and A.Vitturi, Phys. Rev. C 90, 064301-1, 6 (2014).

[5] TUNL, Nuclear data evaluation, http://www.tunl.duke.edu/NuclData/General_Tables/5he.shtml (2009).

[6] T. Myo et al., Progress in Particle and Nuclear Physics 79, 1-56 (2014).

[7] K. Hagino and H. Sagawa, Phys. Rev. C 72, 044321-1, 6 (2005). 